\title{
Reduced 24-h Sodium Excretion Is Associated With a Disturbed Plasma Acylcarnitine Profile in Vasovagal Syncope Children: A Pilot Study
}

\author{
Jinqing Song ${ }^{1 \dagger}$, Chunyan Tao ${ }^{1 \dagger}$, Guozhen Chen ${ }^{1,2}$, Selena Chen ${ }^{3}$, Wenrui $\mathrm{Xu}^{1 *}$, \\ Junbao $D u^{1}$, Yanling Yang ${ }^{1 *}$ and Yaqian Huang ${ }^{1 *}$ \\ ${ }^{1}$ Department of Pediatrics, Peking University First Hospital, Beijing, China, ${ }^{2}$ Department of Pediatrics, The Affiliated Yantai \\ Yuhuangding Hospital of Qingdao University, Yantai, China, ${ }^{3}$ Division of Biological Sciences, University of California, \\ San Diego, La Jolla, CA, United States
}

OPEN ACCESS

Edited by:

Xupei Huang,

Florida Atlantic University,

United States

Reviewed by:

Ju Liu,

Shandong University, China

Cheng Wang,

Central South University, China

${ }^{*}$ Correspondence:

Wenrui Xu

xuwenrui10@126.com

Yanling Yang

yanlinggy@vip.sina.com

Yaqian Huang

yaqianhuang@126.com

†These authors have contributed equally to this work

Specialty section:

This article was submitted to

Pediatric Cardiology

a section of the journal

Frontiers in Pediatrics

Received: 31 December 2019 Accepted: 26 February 2020 Published: 11 March 2020

Citation:

Song J, Tao C, Chen G, Chen S,

$X u W, D u J$, Yang $Y$ and Huang $Y$

(2020) Reduced 24-h Sodium

Excretion Is Associated With a Disturbed Plasma Acylcarnitine Profile in Vasovagal Syncope Children: A Pilot

Study. Front. Pediatr. 8:98.

doi: 10.3389/fped.2020.00098
Objective: To investigate if the low sodium intake is associated with the plasma carnitine and acylcarnitine profile in children with vasovagal syncope (WS).

Materials and Methods: Twenty-six children suffering from WS were recruited in the present study and divided into a group of low urinary sodium excretion or a group of normal urinary sodium excretion according to the excretion of 24-h urinary sodium $<3$ or 3-6g, respectively. The excretion of $24-\mathrm{h}$ urinary sodium was detected with ion-selective electrode approach. Plasma carnitine and acylcarnitine concentrations were measured with tandem mass spectrometry. Each participant completed the head-up tilt test. The demographics, clinical characteristics, hemodynamic parameters and plasma carnitine and acylcarnitine concentrations were compared between the two groups. A bivariate correlation between plasma acylcarnitine profiles and the excretion of 24-h urinary sodium was conducted with Spearman's correlation coefficients.

Results: Of the enrolled WS patients, 14 patients were assigned to the group of low urinary sodium excretion and the remaining 12 patients were assigned to the group of normal urinary sodium excretion. Symptoms of fatigue were more prevalent in the group of low urinary sodium excretion than in the group of normal urinary sodium excretion $(p=0.009)$. Aside from fatigue, no other differences in the demographics, clinical characteristics or hemodynamic parameters during the head-up tilt test were found between the two groups ( $p>0.05)$. Concentrations of plasma tiglylcarnitine (C5:1), hydroxyhexadecanoylcarnitine $(\mathrm{C} 16 \mathrm{OH})$, hydroxyoctadecanoylcarnitine $(\mathrm{C} 18 \mathrm{OH})$, and carnitine C22 were significantly higher in the group of low urinary sodium excretion than in the group of normal urinary sodium excretion (all $p$-values $=0.048$ ); moreover, they were all negatively correlated with 24 -h urinary sodium levels (all $p$-values $=0.016$ ). There were no differences between the two groups in other acylcarnitines or free carnitine.

Conclusions: Reduced excretion of 24-h urinary sodium is associated with a disturbed plasma acylcarnitine profile in children with WS. The findings suggest that restricted sodium intake-induced disturbance of plasma acylcarnitines and related cellular energy metabolism might be involved in the pathogenesis of WS in children.

Keywords: carnitine, acylcarnitine, low sodium intake, vasovagal syncope, children 


\section{INTRODUCTION}

Vasovagal syncope (VVS) is the main form of syncope in children and adolescents and is characterized by syncopal attack and hemodynamic abnormalities during the head-up tilt test (HUTT), including systemic arterial hypotension, bradycardia, or both. It has been reported that VVS accounts for more than $60 \%$ of all pediatric syncopal cases $(1,2)$. Approximately $30 \%$ of affected individuals suffer from recurrent syncopal episodes, which significantly impair their quality of life $(3,4)$. Under such circumstances, efficient interventions are required. However, the exact underlying mechanisms of VVS are not fully understood, which results in an unsatisfactory outcome of treatment to some extent $(5,6)$. Therefore, elucidation of the complex mechanisms of VVS is particularly important. Fatigue, which is related to energy metabolic dysfunction, is common in patients with VVS (7). Moreover, it has been established that skeletal muscle extensively accumulates carnitine and acylcarnitines, which are pivotal in cellular energy production because of their role in transporting long-chain fatty acids from the cellular matrix into the matrix of mitochondrion for subsequent beta-oxidation (8, 9). Nevertheless, it is unclear whether a disturbance of carnitine and acylcarnitine profiles is associated with the pathogenesis of VVS. Low sodium intake is one proposed mechanism of VVS and leads to hypovolemia and alterations in biologically active substances which are likely to contribute to syncopal occurrence, including renin, aldosterone, and catecholamine (10-12). As a result, supplementation of fluid and sodium is a rationally efficient method to treat VVS (13). A possible relationship between the metabolism of carnitine and acylcarnitines and the reducing intake of sodium was mentioned among adults with hypertension (14). However, it is unclear whether low sodium intake in patients with VVS leads to abnormal changes in carnitine and acylcarnitines, which mediates the occurrence of syncope.

Therefore, the present research was undertaken to explore if the low sodium intake is associated with the plasma carnitine and acylcarnitine profile in pediatric VVS patients and discuss the possible underlying mechanisms.

\section{MATERIALS AND METHODS Study Subjects}

Twenty-six patients (19 girls and 7 boys, aged $12.5 \pm 2.7$ years old) with VVS were enrolled in the present research. They visited the Child Syncope Center at Peking University First Hospital from August 2018 to September 2019. All patients underwent an excretion test of 24-h urinary sodium. Based on the excretion levels of 24-h urinary sodium, patients were divided into the group of low urinary sodium excretion (excretion level $<3 \mathrm{~g}$ ) or the group of normal urinary sodium excretion (excretion level between 3 and $6 \mathrm{~g}$ ) (2). No patients were overweight or obese, nor had associated disorders of diabetes mellitus, thyroid dysfunction, renal dysfunction, hepatic dysfunction, or cardiac dysfunction or symptoms and signs of fever, vomiting, diarrhea

Abbreviations: VVS, vasovagal syncope; HUTT, head-up tilt test. or dehydration. The criteria for the diagnosis of pediatric VVS were as follows: (1) syncopal events that were often induced by predisposing factors including rapid postural alterations, longterm standing, and emotional stimulation, etc.; (2) a positive response to the HUTT; as well as (3) excluding other syncopal disorders $(15,16)$. The Ethics Committee of Peking University First Hospital approved this study. We conducted the study in line with the ethical criteria in the Declaration of Helsinki. The legal guardians of each patient signed the informed consent.

\section{Protocol for the HUTT}

All enrolled patients underwent strict inspections to exclude cardiogenic, neurological, and metabolic disorders. Patients then completed the HUTT. This test was conducted in a dimly lit, warm and quiet room between 8:30 and 12:00 a.m. Patients were required to fast and the medication impacting autonomic nervous function was avoided at least 5 half-life times before the HUTT. They were placed on an autonomic stretcher (HUT821; Beijing Juchi, Beijing, China) for 10-20 min to maintain a stable hemodynamic condition. Then, they were positioned at $60^{\circ}$ passively for as long as $45 \mathrm{~min}$ or until a positive response occurred. A positive response to the HUTT was according to the criteria described previously $(15,16)$.

\section{Protocol for the 24-h Urinary Sodium Excretion Test}

Each patient as well as his/her caretakers were introduced to a standard urine collection process in advance. Girls were required to make a collection of 24-h urine samples during non-menstrual periods. The sodium-selective electrode approach (Cobas 6000, Roche, Basel, Switzerland) was done for measuring the excretion of 24-h urinary sodium. The formula is as follows (17): excretion of 24 -h urinary sodium $=$ concentration of sodium $\times$ total $24-\mathrm{h}$ urine volume.

\section{Measurement of Plasma Carnitine and Acylcarnitines}

All VVS patients fasted for at least $4 \mathrm{~h}$ before blood sampling at $\sim 8: 00$ a.m. Two milliliters of blood were drawn by venipuncture and collected in an ethylenediaminetetraacetic acid anticoagulant tube. After being centrifugated at 2,000 $\mathrm{g}$ for $20 \mathrm{~min}$, plasma was extracted at $4^{\circ} \mathrm{C}$ and then preserved at $-80^{\circ} \mathrm{C}$ until tested. The collected plasma was thawed at one time for the measurement of the carnitine and acylcarnitine profile on a tandem mass spectrometer (MS/MS, API3200, Applied Biosystems, California, USA). The concentrations of carnitine and acylcarnitines were calculated automatically using Chemo View software (NeoLynx, Waters, Massachusetts, USA). All the testing work was done by a professionally trained investigator.

\section{Statistical Analysis}

The data were processed with SPSS software 22.0 (IBM Corp., NY, USA). Continuous variables are stated as the mean \pm $\mathrm{SD}$. And between two groups, the differences were measured by Student's $t$-test or Mann-Whitney $U$-test according to the Shapiro-Wilk test consequences. Categorical variables are presented as numbers, and Fisher's exact test was applied for 
TABLE 1 | Baseline characteristics and hemodynamics in the head-up tilt test in vasovagal syncope patients.

\begin{tabular}{|c|c|c|c|c|}
\hline Items & $\begin{array}{l}\text { Low urinary sodium excretion } \\
\text { group }(n=14)\end{array}$ & $\begin{array}{l}\text { Normal urinary sodium excretion } \\
\text { group }(n=12)\end{array}$ & $t / Z / \chi^{2}$ value & $p$-value \\
\hline Gender (n, M/F) & $3 / 11$ & $4 / 8$ & $--^{\dagger}$ & 0.665 \\
\hline Age (years) & $12.1 \pm 3.0$ & $13.0 \pm 2.4$ & -0.788 & 0.439 \\
\hline Body mass index $\left(\mathrm{kg} / \mathrm{m}^{2}\right)$ & $17.6 \pm 2.3$ & $20.0 \pm 3.9$ & -1.916 & 0.067 \\
\hline Symptom duration (months) & $29.6 \pm 37.1^{\star}$ & $17.1 \pm 19.5^{\star}$ & -0.646 & 0.518 \\
\hline Numbers of syncope (times) & $4 \pm 3^{\star}$ & $4 \pm 2^{\star}$ & -1.157 & 0.247 \\
\hline Duration of unconsciousness (min) & $5.2 \pm 8.3^{\star}$ & $1.5 \pm 1.7^{\star}$ & -1.066 & 0.286 \\
\hline Fatigue ( $n$, yes/no) & $13 / 1$ & $5 / 7$ & $--^{\dagger}$ & 0.009 \\
\hline Supine HR (beats/min) & $77 \pm 14^{*}$ & $77 \pm 14$ & -0.180 & 0.857 \\
\hline Supine SBP (mmHg) & $106 \pm 9$ & $110 \pm 11^{*}$ & -1.185 & 0.236 \\
\hline Supine DBP (mmHg) & $62 \pm 6$ & $66 \pm 9$ & -1.070 & 0.295 \\
\hline $\begin{array}{l}\text { Time to positive response in HUTT } \\
\text { (min) }\end{array}$ & $31 \pm 11^{*}$ & $26 \pm 13$ & -1.159 & 0.246 \\
\hline Positive HR in HUTT (beats/min) & $116 \pm 32$ & $114 \pm 16$ & 0.270 & 0.789 \\
\hline Positive SBP in HUTT (mmHg) & $72 \pm 5$ & $71 \pm 5$ & 0.567 & 0.576 \\
\hline Positive DBP in HUTT (mmHg) & $48 \pm 7^{\star}$ & $48 \pm 4$ & -0.438 & 0.661 \\
\hline
\end{tabular}

HR, heart rate; SBP, systolic blood pressure; DBP, diastolic blood pressure; HUTT, head-up tilt test; Data are mean \pm SD or numbers.

*Non-normality.

${ }^{\dagger}$ Fisher's exact test.

comparison. A bivariate correlation between data with nonnormal distribution was conducted with Spearman's correlation coefficients. Significance was considered when $p$-value $<0.05$.

\section{RESULTS}

\section{Basic Information of VVS Patients}

Among the enrolled VVS patients, the group of low urinary sodium excretion included 14 patients with a 24-h urinary sodium excretion of $0.8-2.7 \mathrm{~g}$ (mean $2.1 \pm 0.6 \mathrm{~g}$ ), and the group of normal urinary sodium excretion included 12 children with a 24-h urinary sodium excretion of 3.1-5.9 g (mean $3.8 \pm 0.8 \mathrm{~g}$ ). No differences existed in the demographics, and clinical and hemodynamic characteristics between the two groups $(p>0.05$, Table 1). However, more children in the group of low urinary sodium excretion reported fatigue than in the group of normal urinary sodium excretion ( $p=0.009$, Table 1 ).

\section{Plasma Carnitine and Acylcarnitine Profile in VVS Patients}

Levels of several acylcarnitines, namely tiglylcarnitine (C5:1) $(p=0.048)$, hydroxyhexadecanoylcarnitine $(\mathrm{C} 16 \mathrm{OH})(p=$ $0.048)$, hydroxyoctadecanoylcarnitine $(\mathrm{C} 18 \mathrm{OH})(p=0.048)$, and carnitine $\mathrm{C} 22$ ( $p=0.048$ ), were higher in the group of low urinary sodium excretion than in the group of normal urinary sodium excretion (Table 2). No statistically significant differences were showed between the two groups in other acylcarnitines or free carnitine $(\mathrm{C} 2, p=0.504$; $\mathrm{C} 3, p=0.777$; $\mathrm{C} 3 \mathrm{DC}, p=0.803$; $\mathrm{C} 4, p$ $=0.620$; 5 , $p=1.000$; C5DC, $p=0.912$; $50 H, p=0.654$; C6, $p=0.312$; C6DC, $p=0.354$; $8, p=0.288$; $88: 1, p=0.313$; $\mathrm{C} 10$, $p=0.359 ; \mathrm{C} 10: 1, p=0.086 ; \mathrm{C} 10: 2, p=0.192 ; \mathrm{C} 12, p=0.720$; $\mathrm{C} 12: 1, p=0.083 ; \mathrm{C} 14, p=0.845 ; \mathrm{C} 14: 1, p=0.565 ; \mathrm{C} 14: 2, p=$
0.146; C14DC, $p=0.181$; C14OH, $p=0.234$; C16, $p=0.897$; $\mathrm{C} 16: 1, p=0.863 ; \mathrm{C} 16: 1 \mathrm{OH}, p=0.725 ; \mathrm{C} 18, p=0.958 ; \mathrm{C} 18: 1, p$ $=0.813$; $\mathrm{C} 18: 1 \mathrm{OH}, p=0.481$; $18: 2, p=0.813$; C20, $p=0.105$; $\mathrm{C} 24, p=0.457$; $226, p=0.355$; 5 /C2, $p=0.297$; $2 / \mathrm{C} 0, p=$ 0.366 ; $\mathrm{C} 3 / \mathrm{C} 2, p=0.602$; $\mathrm{C} 3 / \mathrm{C} 0, p=1.000$; 8 /C2, $p=0.867$; C14:1/C16, $p=0.909$; free carnitine, $p=0.897$, Table 2 ).

\section{Correlations Between the Excretion of 24-h Urinary Sodium and Plasma Acylcarnitines}

Further examining the association between the excretion levels of 24-h urinary sodium and the abovementioned plasma acylcarnitines with significant difference, negative Spearman's correlation coefficients were found (C5:1, $r=-0.469, p=0.016$; $\mathrm{C} 16 \mathrm{OH}, r=-0.469, p=0.016$; $\mathrm{C} 18 \mathrm{OH}, r=-0.469, p=0.016$; and $\mathrm{C} 22, r=-0.469, p=0.016)$ in all study patients.

\section{DISCUSSION}

In mitochondria, carnitine and acylcarnitines are a series of essential molecules that play an obligatory part in the long-chain fatty acid beta-oxidation. Analyzing carnitine and acylcarnitine profile may help in the investigation and elucidation of metabolic derangements. The acylcarnitine profile displays a characteristic alteration in inherited metabolic diseases, such as short chain and medium chain acyl coenzyme A dehydrogenase deficiency, and carnitine acylcarnitine translocase deficiency (18). Moreover, it was reported that changes in the acylcarnitine profile were related to the development of IgA nephropathy and diabetes mellitus $(19,20)$. In the study, we observed that in VVS patients, some plasma acylcarnitines $(\mathrm{C} 5: 1, \mathrm{C} 16 \mathrm{OH}, \mathrm{C} 18 \mathrm{OH}, \mathrm{C} 22)$ were higher in the group of low urinary sodium excretion than in the group of normal 
TABLE 2 | Plasma carnitine and acylcarnitines in vasovagal syncope patients.

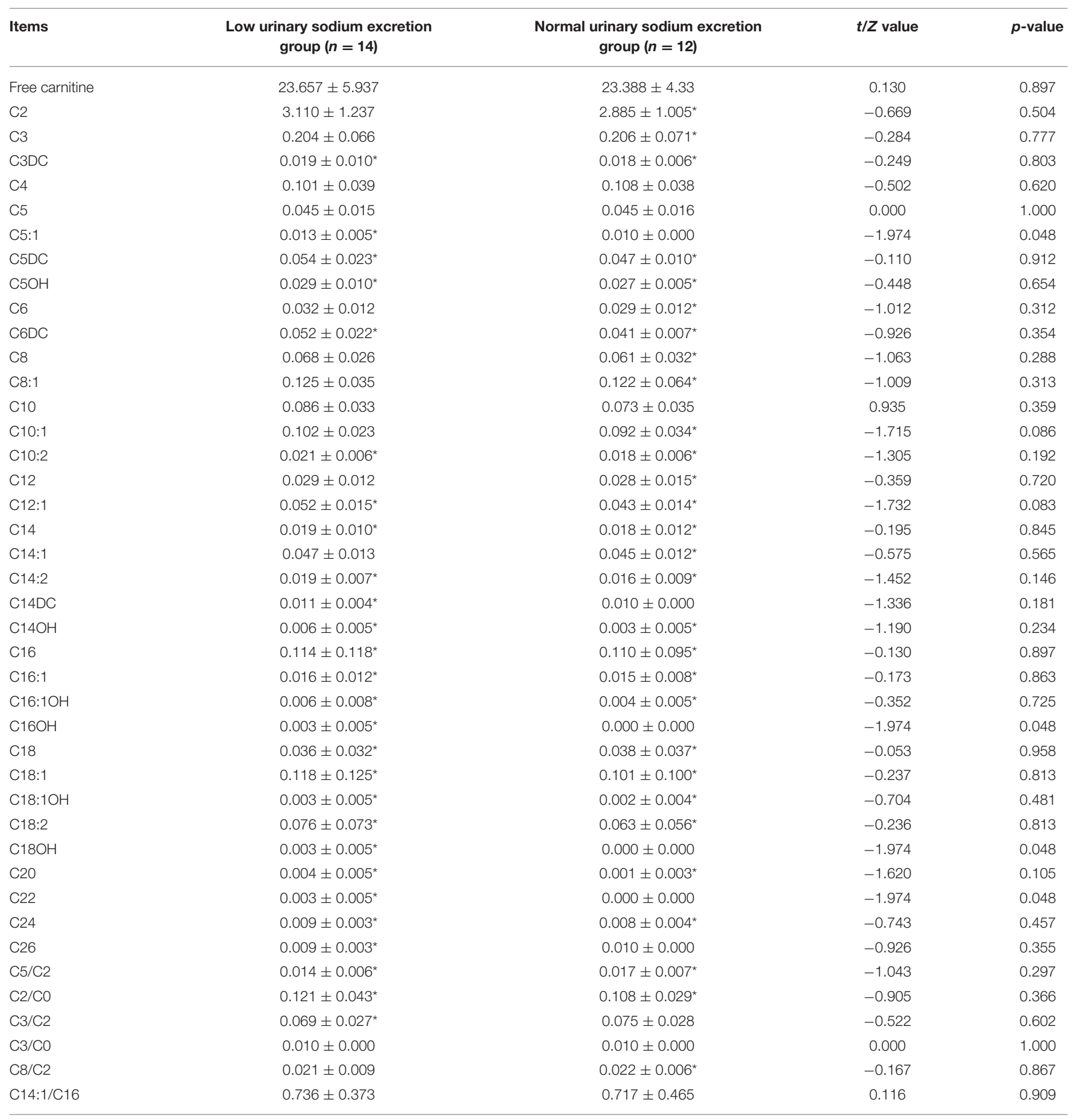

Data are mean $\pm S D$.

*Non-normality.

urinary sodium excretion. Moreover, the excretion of 24-h urinary sodium was negatively correlated with these plasma acylcarnitines. Patients suffering from VVS with low urinary sodium excretion were more susceptible to experiencing symptoms of fatigue than those in the group of normal urinary sodium excretion.
The mechanisms by which low sodium loading results in a disturbed acylcarnitine profile have not been explored. Derkach et al. also found a phenomenon in a DASH-sodium trial among participants with high blood pressure, that plasma metabolites of acylcarnitines (butyrylcarnitine and valerylcarnitine) were increased with sodium reduction (14). On the other hand, 
some studies showed a significant increase in renin-angiotensinaldosterone system activity with low sodium intake (12). The activated renin-angiotensin-aldosterone system is capable of inducing oxidative stress $(21,22)$, which could diminish the activity of enzymes in metabolic processes. Therefore, we inferred that low sodium loading might impair the activity of enzymes involved in the long-chain fatty acid beta-oxidation. The inefficient long-chain fatty acid beta-oxidation could promote the accumulation of acylcarnitine byproducts from substrate catabolism, which are capable of passing across the mitochondrial membrane and the cell membrane $(19,23,24)$. More convincingly, 24-h urinary sodium excretion was found to be negatively correlated with the concentration of several acylcarnitines in our study, which suggested that reduced dietary sodium intake might be associated with abnormal fatty acid metabolism.

As a high-energy-consuming organ, skeletal muscle needs fatty acids to supplement essential energy during exercise or stress (25). Insufficient energy leads to fatigue. Large numbers of VVS patients complain of fatigue before or after syncope occurrence (7). In our Syncope Unit, we also observed several patients experiencing fatigue for long durations of time. In the present study, patients with low urinary sodium excretion were more prone to experience fatigue than those with normal urinary sodium excretion. This highlights that some VVS patients are disturbed by energy metabolism dysfunction, which is likely induced, at least in part, by low sodium intake.

The excretion of 24-h urinary sodium, a standard criterion for the measurement of sodium intake (26), is applied in many investigations to explore the relationship between sodium intake and development of diverse disorders. Kieneker et al. conducted a study in 7,330 individuals and found a strong relationship between low urinary sodium level and an increased stroke risk (27). Moreover, many investigations have showed that abnormal sodium intake is related to increased mortality and cardiovascular events $(28,29)$. The relationship between sodium intake and outcomes of health should be a U-shape, where high and low sodium intake indicated a risk factor for negative outcomes. We suggested that low sodium intake might decrease the long-chain fatty acid beta-oxidation rate and increase the plasma concentration of acylcarnitines in VVS patients. Additional and longer investigations should be performed to determine whether low sodium intake can predict the outcomes of affected individuals.

In summary, increased plasma concentration of acylcarnitines in VVS patients with low urinary sodium excretion was reported for the first time. In addition to VVS, postural tachycardia syndrome is another subtype of orthostatic intolerance with fatigue, palpitation and dizziness as well as syncope in the childhood (30, 31). Furthermore, some postural tachycardia

\section{REFERENCES}

1. Romme JJ, van Dijk N, Boer KR, Dekker LR, Stam J, Reitsma JB, et al. Influence of age and gender on the occurrence and presentation of reflex syncope. Clin Auton Res. (2008) 18:127-33. doi: 10.1007/s10286-008-0 465-0 syndrome patients are also characterized with low sodium intake $(32,33)$. As such, we speculate that the restricted sodium intakeinduced disturbance of plasma acylcarnitines and cellular energy metabolism might be associated with the pathogenesis of not only VVS, but also other subtypes of orthostatic intolerance in children, which merits further investigations. Also, there were some limitations, such as a small sample size, the singlecenter observational study design and the lack of healthy control participants. Future multicenter-based, larger samplesized and mechanistic studies should be performed to explore the significance of low-sodium-intake-associated energy metabolism in pathogenesis of VVS.

\section{DATA AVAILABILITY STATEMENT}

The datasets generated for this study are available on request to the corresponding author.

\section{ETHICS STATEMENT}

The studies involving human participants were reviewed and approved by the Ethics Committee of Peking University First Hospital. Written informed consent to participate in this study was provided by the participants' legal guardian/next of kin.

\section{AUTHOR CONTRIBUTIONS}

JS had the primary responsibility for the protocol development, examined the plasma concentrations of carnitine and acylcarnitines. CT was responsible for patient enrollment, data collection, and preliminary data analysis. JS and CT wrote the drafted manuscript. GC, SC, JD, and WX assisted with the data collection and data analysis. GC, JD, and SC revised the manuscript and gave important advice on the subject. WX, YY, and YH supervised the design and execution of this study, checked data analysis, revised the manuscript, and had a final approval of the submitted manuscript. All authors have read and approved the final manuscript and assumed full responsibility for its contents.

\section{FUNDING}

This study was supported by Peking University Clinical Scientist Program (BJMU2019LCKXJ001) and the Fundamental Research Funds for the Central Universities.

\section{ACKNOWLEDGMENTS}

The authors thank all the children as well as their caretakers for participating in this study.

2. Alderman MH. Dietary sodium: where science and policy diverge. Am J Hyperten. (2016) 29:424-7. doi: 10.1093/ajh/hpu256

3. Ng J, Sheldon RS, Ritchie D, Raj V, Raj SR. Reduced quality of life and greater psychological distress in vasovagal syncope patients compared to healthy individuals. Pacing Clin Electrophysiol. (2019) 42:1808. doi: $10.1111 /$ pace. 13559 
4. Anderson JB, Czosek RJ, Knilans TK, Marino BS. The effect of paediatric syncope on health-related quality of life. Cardiol Young. (2012) 22:5838. doi: $10.1017 /$ S1047951112000133

5. Vyas A, Swaminathan PD, Zimmerman MB, Olshansky B. Are treatments for vasovagal syncope effective? A meta-analysis. Int J Cardiol. (2013) 167:190611. doi: 10.1016/j.ijcard.2012.04.144

6. Romano S, Branz L, Fondrieschi L, Minuz P. Does a therapy for refex vasovagal syncope really exist? High Blood Press Cardiolvasc Prev. (2019) 26:273-82. doi: 10.1007/s40292-019-00327-3

7. Legge $H$, Norton $M$, Newton JL. Fatigue is significant in vasovagal syncope and is associated with autonomic symptoms. Europace. (2008) 10:1095101. doi: 10.1093/europace/eun164

8. Almannai M, Alfadhel M, El-Hattab AW. Carnitine inborn errors of metabolism. Molecules. (2019) 24:E3251. doi: 10.3390/molecules24183251

9. Tamai I, Ohashi R, Nezu J, Yabuuchi H, Oku A, Shimane M, et al. Molecular and functional identification of sodium ion-dependent, high affinity human carnitine transporter OCTN2. J Biol Chem. (1998) 273:2037882. doi: 10.1074/jbc.273.32.20378

10. Mosqueda-Garcia R, Furlan R, Tank J, Fernandez-Violante R. The elusive pathophysiology of neurally mediated syncope. Circulation. (2000) 102:2898906. doi: 10.1161/01.CIR.102.23.2898

11. El-Sayed H, Hainsworth R. Salt supplement increases plasma volume and orthostatic tolerance in patients with unexplained syncope. Heart. (1996) 75:134-40. doi: 10.1136/hrt.75.2.134

12. Graudal NA, Hubeck-Graudal T, Jurgens G. Effects of low sodium diet versus high sodium diet on blood pressure, renin, aldosterone, catecholamines, cholesterol, and triglyceride. Cochrane Database Syst Rev. (2011) doi: 10.1002/14651858.CD004022.pub3.

13. Chu W, Wang C, Wu L, Lin P, Li F, Zou R. Oral rehydration salts: an effective choice for the treatment of children with vasovagal syncope. Pediatr Cardiol. (2015) 36:867-72. doi: 10.1007/s00246-015-1097-5

14. Derkach A, Sampson J, Joseph J, Playdon MC, Stolzenberg-Solomon RZ. Effects of dietary sodium on metabolites: the Dietary Approaches to Stop Hypertension (DASH)-Sodium Feeding Study. Am J Clin Nutr. (2017) 106:1131-41. doi: 10.3945/ajcn.116.150136

15. Tao C, Chen S, Li H, Wang Y, Wang Y, Liu P, et al. Value of immediate heart rate alteration from supine to upright in differential diagnosis between vasovagal syncope and postural tachycardia syndrome in children. Front Pediatr. (2018) 6:343. doi: 10.3389/fped.2018.00343

16. Wang C, Li Y, Liao Y, Tian H, Huang M, Dong XY, et al. 2018 Chinese Pediatric Cardiology Society (CPCS) guideline for diagnosis and treatment of syncope in children and adolescents. Sci Bull. (2018) 63:155864. doi: 10.1016/j.scib.2018.09.019

17. Tao C, Lu W, Lin J, Li H, Li X, Tang C, et al. Long-term outcomes of children and adolescents with postural tachycardia syndrome after conventional treatment. Front Pediatr. (2019) 7:261. doi: 10.3389/fped.2019.00261

18. Peng L, Liu L, Peng M, Jiang M. Application of acylcarnitine analysis on the screening and diagnosis of inherited metabolic diseases. J Appl Clin Pediatr. (2012) 27:1617-20. doi: 10.3969/j.issn.1003-515X.2012.20.025

19. Zhang X, Zhang C, Chen L, Han X, Ji L. Human serum acylcarnitine profiles in different glucose tolerance states. Diabetes Res Clin Pract. (2014) 104:37682. doi: 10.1016/j.diabres.2014.04.013

20. Xia F, Zhu L, Xu C, Wu Q, Chen W, Zeng R, et al. Plasma acylcarnitines could predict prognosis and evaluate treatment of IgA nephropathy. Nutr Metab (Lond). (2019) 16:2. doi: 10.1186/s12986-018-0328-1
21. Münzel T, Gori T, Keaney JF Jr, Maack C, Daiber A. Pathophysiological role of oxidative stress in systolic and diastolic heart failure and its therapeutic implications. Eur Heart J. (2015) 36:2555-64. doi: 10.1093/eurheartj/ehv305

22. Haas MJ, Onstead-Haas L, Lee T, Torfah M, Mooradian AD. Angiotensin II receptor one (AT1) mediates dextrose induced endoplasmic reticulum stress and superoxide production in human coronary artery endothelial cells. Int J Cardiol. (2016) 220:842-50. doi: 10.1016/j.ijcard.2016.06.094

23. Koves TR, Ussher JR, Noland RC, Slentz D, Mosedale M, Ilkayeva $\mathrm{O}$, et al. Mitochondrial overload and incomplete fatty acid oxidation contribute to skeletal muscle insulin resistance. Cell Metab. (2008) 7:4556. doi: 10.1016/j.cmet.2007.10.013

24. Adams SH, Hoppel CL, Lok KH, Zhao L, Wong SW, Minkler PE, et al. Plasma acylcarnitine profiles suggest incomplete long-chain fatty acid beta-oxidation and altered tricarboxylic acid cycle activity in type 2 diabetic AfricanAmerican women. J Nutr. (2009) 139:1073-81. doi: 10.3945/jn.108.103754

25. Lu Km, Nishimori H, Nakamura Y, Shima K, Kuwajima M. A missense mutation of mouse OCTN2, a sodium-dependent carnitine cotransporter, in the juvenile visceral steatosis mouse. Biochem Biophys Res Commun. (1998) 252:590-4. doi: 10.1006/bbrc. 1998.9708

26. McLean RM. Measuring population sodium intake: a review of methods. Nutrients. (2014) 6:4651-62. doi: 10.3390/nu6114651

27. Kieneker LM, Eisenga MF, Gansevoort RT, de Boer RA, Navis G, Dullaart $\mathrm{RPF}$, et al. Association of low urinary sodium excretion with increased risk of stroke. Mayo Clin Proc. (2018) 93:1803-9. doi: 10.1016/j.mayocp.2018. 05.028

28. Graudal N, Jürgens G, Baslund B, Alderman MH. Compared with usual sodium intake, low- and excessive-sodium diets are associated with increased mortality: a meta-analysis. Am J Hypertens. (2014) 27:112937. doi: 10.1093/ajh/hpu028

29. O’Donnell M, Mente A, Rangarajan S, McQueen MJ, Wang X, Liu L, et al. Urinary sodium and potassium excretion, mortality, and cardiovascular events. N Engl J Med. (2014) 317:612-23. doi: 10.1056/NEJMoa1311889

30. Goodman BP. Evaluation of postural tachycardia syndrome (POTS). Auton Neurosci. (2018) 215:12-9. doi: 10.1016/j.autneu.2018.04.004

31. Bryarly M, Phillips LT, Fu Q, Vernino S, Levine BD. Postural orthostatic tachycardia syndrome: JACC focus seminar. J Am Coll Cardiol. (2019) 73:1207-28. doi: 10.1016/j.jacc.2018.11.059

32. Zhang Q, Liao Y, Tang C, Du J, Jin H. Twenty-four-hour urinary sodium excretion and postural orthostatic tachycardia syndrome. J Pediatr. (2012) 161:281-4. doi: 10.1016/j.jpeds.2012.01.054

33. Mar PL, Raj SR. Postural orthostatic tachycardia syndrome: mechanisms and new therapies. Annu Rev Med. (2020) 71:23548. doi: 10.1146/annurev-med-041818-011630

Conflict of Interest: The authors declare that the research was conducted in the absence of any commercial or financial relationships that could be construed as a potential conflict of interest.

Copyright (c) 2020 Song, Tao, Chen, Chen, Xu, Du, Yang and Huang. This is an open-access article distributed under the terms of the Creative Commons Attribution License (CC BY). The use, distribution or reproduction in other forums is permitted, provided the original author(s) and the copyright owner(s) are credited and that the original publication in this journal is cited, in accordance with accepted academic practice. No use, distribution or reproduction is permitted which does not comply with these terms. 